\title{
A COMBINED COSMOLOGICAL TEST
}

\author{
J.-E. SOLHEIM \\ Institute of Theoretical Astrophysics, Oslo, Norway*
}

\begin{abstract}
I shall report the result of a combined cosmological test, including both galaxies in clusters and blue objects of different types. The test is performed on uniform models and the result is a high density, ever expanding model.
\end{abstract}

The data set includes B and V magnitudes for galaxies in 21 clusters, the old data from HMS and Baum, 10 Seyfert galaxies of spiral type (SYS), 6 Seyfert galaxies of $\mathrm{N}$-type together with $8 \mathrm{~N}$ galaxies, 3 compact galaxies, 23 Radio Quiet blue objects, where those with small redshift $(z<0.5)$, called RQ I, are treated separately, and finally a sample of 102 Quasi Stellar Radio Sources (QSRS). Magnitudes and redshifts are taken from various sources (Krieger and Solheim, 1971), most from a list compiled by Barbieri (1969). For the RQs and the QSRS the spread in the data is somewhat reduced by using mean values of 3 or 7 objects grouped together in $z$. Whenever possible the $K$-corrections have been applied to the data, but no evolutionary corrections are applied.

The test is performed with a program developed by Solheim (1966), giving the best fit among uniform models for a given data set. The program is generalized to take care of several classes of objects with different absolute magnitudes. The result is shown in the model diagram (Figure 1). The best fit when both the $\mathrm{B}$ and $\mathrm{V}$ magnitudes are used together, is the model with $q_{0}=1.1$ and $\sigma_{0}=8.1$, which is a rather high density model of the ever expanding type (Stabell and Refsdal, 1966). The density determined here is much higher than in the model determined from the newer photoelectric measurements of galaxies in clusters (Peach, 1970, 1971) but may be changed considerably when evolutionary corrections are applied.

The $64 \%$ confidence area found in this test is rather small, and this is due to the large number of observations included. Altogether we have used about 400 observations which give a fit to the model with a probable error of 0.17 mag.

These formal results are not too realistic till we know more about the objects used in the test. One thing we can do is to use the model determined to calibrate the data in absolute magnitudes and colors. Such a calibration is shown in Figure 2 which gives $M_{B}$ vs $(\mathrm{B}-\mathrm{V})_{0}$ for the 7 classes of objects tested.

The objects seem to define a red branch and a blue branch - with the RQ I objects (Radio Quiets with small redshift) in an intermediate position. A similar test for the U-magnitudes shows that the RQs and QSRS have about the same $(\mathrm{U}-\mathrm{B})_{0}$ color. From this we may conclude that we have $t w o$ classes of Radio Quiet objects with somewhat different properties as shown in Table I.

(I) Nearby objects $(z<0.5)$ which may be true non-radio sources. They have

* Present address, University of Tromsö, Norway. 


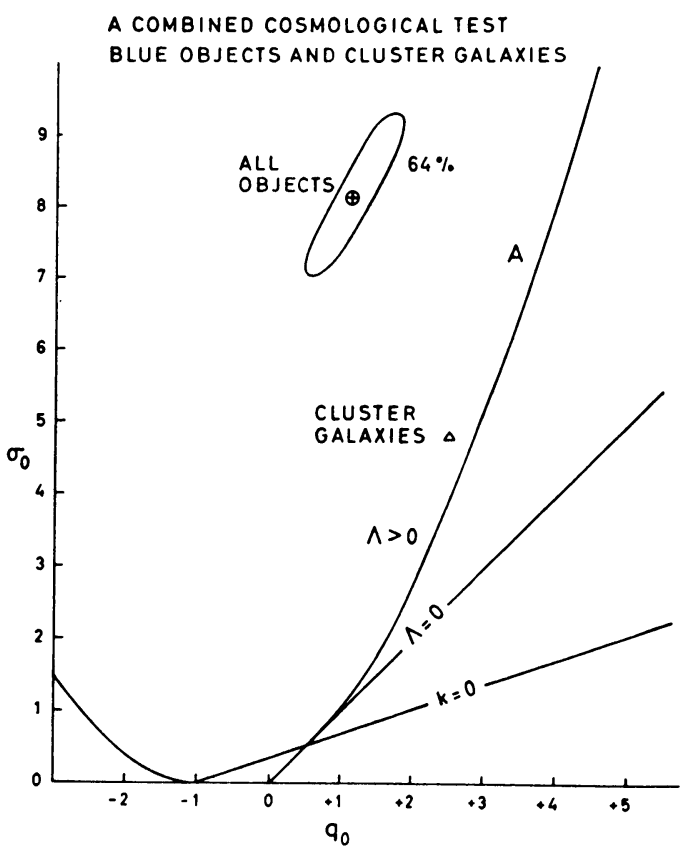

Fig. 1.

ABSOLUTE MAGNITUDES

AND COLORS IN MODEL

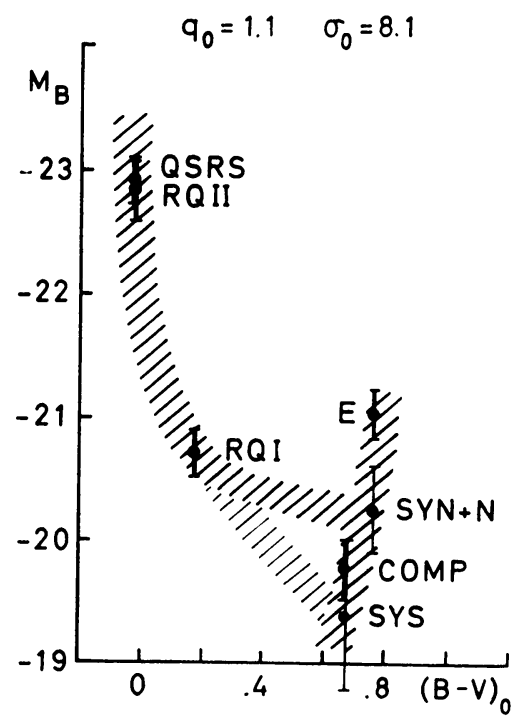

Fig. 2. 
TABLE I

Absolute magnitudes and colors

\begin{tabular}{lccc} 
& Model: $\mathrm{q}_{0}=1.1, \sigma_{0}=8.1, H_{0}=100 \mathrm{~km} \mathrm{~s}^{-1} \mathrm{Mpc}^{-1}$ \\
\hline Type & $M_{B}$ & $(\mathrm{~B}-\mathrm{V})_{0}$ & $(\mathrm{U}-\mathrm{B})_{0}$ \\
\hline RQ I & -20.7 & 0.16 & -0.80 \\
RQ II & -22.8 & -0.03 & -0.76 \\
QSRS & -22.9 & -0.04 & -0.84 \\
\hline
\end{tabular}

optical magnitudes 2 mag. fainter than the QSRS and somewhat redder in $(\mathrm{B}-\mathrm{V})_{0}$.

(II) Remote objects $(z>1.2)$ which have the same optical magnitudes and colors as QSRS - but may have radio-emission too weak to be observed.

When this test was done no Radio Quiet object was known with redshift between 0.5 and 1.2. The results indicate that RQ I objects should not be used in cosmological tests together with the QSRS without correction for the large magnitude difference.

Finally the objects seem to define a sequence which may be an indication of some kind of evolution from a Quasi Stellar source to ordinary galaxies with the RQ I and the Seyfert galaxies and the $\mathrm{N}$-type as the different steps in between.

\section{References}

Barbieri, C.: 1969, Revised Catalogue of Quasistellar Objects, private communication, April. Krieger, C. J. and Solheim, J.-E.: 1971, to be published.

Peach, J.: 1970, Astrophys. J. 159, 753.

Peach, J.: 1971, this volume, p. 314.

Solheim, J.-E.: 1966, Monthly Notices Roy. Astron. Soc. 133, 321.

Stabell, R. and Refsdal, S.: 1966, Monthly Notices Roy. Astron. Soc. 132, 379.

\section{Discussion}

Barnothy: With $\sigma=8.1$ and $H=75 \mathrm{~km} \mathrm{~s}^{-1} \mathrm{Mpc}^{-1}$ the density of matter in your model is 270 times higher than the observed density of visible matter $\left(7 \times 10^{-31} \mathrm{gm} \mathrm{cm}^{-3}\right)$. While a factor of 20 may be explained by the existence of dead galaxies in clusters of galaxies, I wonder how you explain your high density?

Solheim: Highest weight in this determination of the density is given to the magnitudes and redshifts of the quasi-stellar radio sources, and I have used all sources known one year ago without any rejection. This obviously leads to serious selection effects, because we include too many faint QSOs with small redshifts. If we correct for this luminosity effect, we may reduce the density. 